Article

\title{
Identification of Dehydroxytrichostatin $A$ as a Novel Up-Regulator of the ATP-Binding Cassette Transporter A1 (ABCA1)
}

\author{
Yang $\mathrm{Xu}^{\dagger}$, Yanni Xu ${ }^{\dagger}$, Yi Bao, Bin Hong * and Shuyi Si * \\ Institute of Medicinal Biotechnology, Peking Union Medical College and Chinese Academy of \\ Medical Sciences, Beijing 100050, China \\ $\dagger$ These authors contributed equally to this work. \\ * Authors to whom correspondence should be addressed; E-Mails: sisyimb@hotmail.com (S.S.); \\ binhong69@hotmail.com (B.H.); Tel.: +86-10-63180604 (S.S.); +86-10-63028003 (B.H.); \\ Fax: +86-10-63180604 (S.S.); +861063017302 (B.H.).
}

Received: 25 May 2011; in revised form: 24 June 2011 / Accepted: 25 July 2011 /

Published: 25 August 2011

\begin{abstract}
The ATP-binding cassette transporter A1 (ABCA1) mediates the cellular efflux of excess cholesterol and phospholipids to lipid-poor apolipoprotein A-I (apoA-I). ABCA1 plays an important role in high-density lipoprotein (HDL) biogenesis and reverse cholesterol transport. By using a cell-based screening model for the ABCA1 up-regulator and column chromatography, an active compound, 9179B, was isolated. Through analysis of its NMR data, 9179B was identified as dehydroxytrichostatin A. We found that 9179B increased the transcription of ABCA1 in a cell-based reporter assay, with an $\mathrm{EC}_{50}$ value of $2.65 \mu \mathrm{M}$. 9179B up-regulated ABCA1 expression at both mRNA and protein levels in HepG2 and RAW264.7 cells. It also up-regulated the expression of scavenger receptor class B type I (SR-BI) as well as the uptake of DiI-HDL in RAW264.7 cells. This compound stimulated ApoA-I-mediated cellular cholesterol efflux from RAW 264.7 cells. We further found that 9179B was a potent histone deacetylase (HDAC) inhibitor with an $\mathrm{IC}_{50}$ value of $0.08 \mu \mathrm{M}$. Reporter gene assays showed that the regulation of ABCA1 transcription by $9179 \mathrm{~B}$ was mainly mediated by the $-171 /-75$ bp promoter region. Together, our results indicate that $9179 \mathrm{~B}$ is an ABCA1 up-regulator and dehydroxytrichostatin A may be a novel anti-atherogenic compound.
\end{abstract}


Keywords: dehydroxytrichostatin A; atherosclerosis; ABCA1; up-regulator; HDAC inhibitor

\section{Introduction}

Cardiovascular disease (CVD) is a major threat to human health and atherosclerosis (AS) is the principal pathogenesis of CVD. AS is a chronic inflammatory disease that is triggered by the build-up of cholesterol-rich plaques in the intima of arteries [1]. Consistently, epidemiological studies have shown a strong inverse relationship between plasma high-density lipoprotein cholesterol (HDL-C) levels and the incidences of CVD [2,3]. Other studies demonstrate that low total cholesterol and high HDL-C levels are associated with low morbidity and mortality rates of CVD [4]. Considerable evidence shows that therapeutically elevating HDL-C levels may be beneficial for CVD.

The ATP-binding cassette transporter A1 (ABCA1) is a membrane transporter, which is highly expressed in placenta, brain, adrenal glands and liver [5]. It mediates the rate-controlling step in HDL particle formation as well as the efflux of cellular phospholipids and cholesterol to lipid-free apolipoprotein A-I (apoA-I), which may account for the atheroprotective effect of HDL [6,7]. The high basal expression of ABCA1 in hepatocytes facilitates the synthesis of pre-beta-HDL and promotes the efflux of cellular phospholipids and cholesterol to lipid-free apoA-I to form nascent HDL [8]. Furthermore, ABCA1 in the liver and intestine contributes to $80 \%$ and $20 \%$ of the HDL biogenesis, respectively [9]. Overexpression of hepatic ABCA1 raises HDL-C levels considerably [15-17]. Mutations of the ABCA1 gene can result in Tangier disease, a severe HDL deficiency characterized by the accumulation of cholesterol in tissues enriched in macrophages [10,11]. Increased ABCA1 expression in human macrophages prevents arterial inflammation [12]. The importance of ABCA1 in HDL metabolism and its atheroprotective effects have been confirmed in apoE knockout mice and in C57BL/6 mice with diet-induced AS $[13,14]$. Thus, ABCA1 could be a promising therapeutic target for CVD.

In our previous study, a high-throughput screening (HTS) method for ABCA1 up-regulators has been developed [18]. In the present study, 2,600 crude microbial secondary metabolite extracts were screened. Strain IA04-9179 is a positive hit in the HTS assay and the extract from this strain displays a potent activity in upregulating the transcription of ABCA1. In this report, we identify $9179 \mathrm{~B}$ as the active compound produced by strain IA04-9179. The chemical structure of 9179B was identified as dehydroxytrichostatin A by NMR. Dehydroxytrichostatin A inhibits histone deacetylase (HDAC) and increases ABCA1 expression in HepG2 and RAW 264.7 cells. Furthermore, treatment of HepG2 cells with 9179B and the cis-elements in ABCA1 promoter up-regulates ABCA1 expression.

\section{Results and Discussion}

\subsection{Isolation and Identification of $9179 B$ from Strain 04-9179}

Strain 04-9179 increased the luciferase activity of ABCA1p-LUC HepG2 cells. After the purification through column chromatography with HP20 macroporous resin, ODS reverse-phase column, and semipreparative HPLC, an active compound named 9179B was isolated. The UV 
spectrum of $9179 \mathrm{~B}$ in methanol showed a $\lambda_{\max }$ of 266 and $350 \mathrm{~nm}$. The purified compound was identified by ESI, FAB, ${ }^{1} \mathrm{H}-\mathrm{NMR},{ }^{13} \mathrm{C}-\mathrm{NMR}$, and $2 \mathrm{D}$ NMR. The molecular formula of $9179 \mathrm{~B}$ was established to be $\mathrm{C}_{17} \mathrm{H}_{22} \mathrm{~N}_{2} \mathrm{O}_{2}$ by MS and NMR. The structure of $9179 \mathrm{~B}$ is similar to trichostatin $\mathrm{A}$ by ${ }^{13} \mathrm{C}-\mathrm{NMR}$ (Table 1). The chemical shift of the olefin carbon (C-2) was decreased in 9179B, which suggests a different substituent group at the C-1 carbonyl carbon. The ESI and HMBC results showed that for 9179B, a hydrogen atom is substituted for the hydroxyl group in the hydroxamic acid of trichostatin A. Therefore, the structure of $9179 \mathrm{~B}$ was identified as the trichostatin derivative 7-(4-(dimethylamino)phenyl)-4,6-dimethyl-7-oxohepta-2,4-dienamide (Figure 1) or dehydroxytrichostatin A.

Table 1. ${ }^{1} \mathrm{H}-\mathrm{NMR}$ and ${ }^{13} \mathrm{C}-\mathrm{NMR}$ data of the compound, 9179B.

\begin{tabular}{|c|c|c|}
\hline Position & ${ }^{13}$ C-NMR ${ }^{a}$ & ${ }^{1}$ H-NMR ${ }^{b}$ \\
\hline 1 & n.d. ${ }^{\mathrm{c}}$ & \\
\hline 2 & 120.1 & $5.97(1 \mathrm{H}, \mathrm{d}, J=15.5)$ \\
\hline 3 & 146.9 & $7.10(1 \mathrm{H}, \mathrm{d}, J=15.5)$ \\
\hline 4 & 134.3 & \\
\hline 5 & 141.1 & $5.86(1 \mathrm{H}, \mathrm{d}, J=9.5)$ \\
\hline 6 & 41.6 & $4.48(1 \mathrm{H}, \mathrm{m})$ \\
\hline 7 & 201.4 & \\
\hline 8 & 124.6 & \\
\hline 9,13 & 131.8 & $7.81(2 \mathrm{H}, \mathrm{d}, J=9.0)$ \\
\hline 10,12 & 111.9 & $6.67(2 \mathrm{H}, \mathrm{d}, J=9.0)$ \\
\hline 11 & 155.4 & \\
\hline 4-Me & 12.7 & $1.80(3 \mathrm{H}, \mathrm{s})$ \\
\hline 6-Me & 18.2 & $1.22(3 \mathrm{H}, \mathrm{d}, J=6.5)$ \\
\hline $\mathrm{N}-\mathrm{Me}_{2}$ & 40.2 & $3.02(6 \mathrm{H}, \mathrm{s})$ \\
\hline
\end{tabular}

Figure 1. The chemical structure of 9179B.<smiles>CC(=C/C(C)C(=O)c1ccc(N(C)C)cc1)/C=C/C(N)=O</smiles>

\subsection{Effect of $9179 B$ and Other HDAC Inhibitors on ABCA1 Promoter Activity}

To determine the bioactivity of $9179 \mathrm{~B}$ on the transcriptional activation of the ABCA1 gene promoter, a dose-response curve of luciferase activity was determined in ABCA1p-LUC HepG2 cells. 9179B increased the luciferase activity in ABCA1p-LUC HepG2 cells in a dose-dependent manner, with an $\mathrm{EC}_{50}$ value of $2.65 \mu \mathrm{M}$. 9179B strongly induced the transcription driven by the ABCA1 promoter, reaching more than 3-fold at $6.99 \mu \mathrm{M}$ (Figure 2A). 
Figure 2. The effect of 9179B and other HDAC inhibitors on ABCA1 promoter activity.

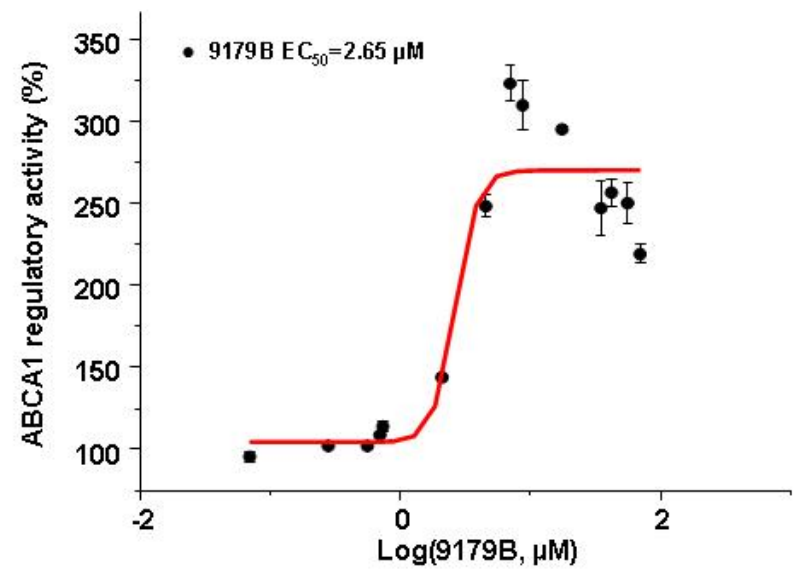

(A)

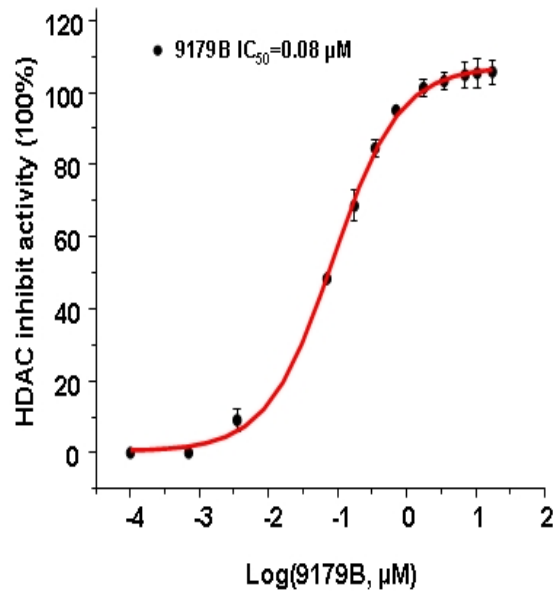

(B)

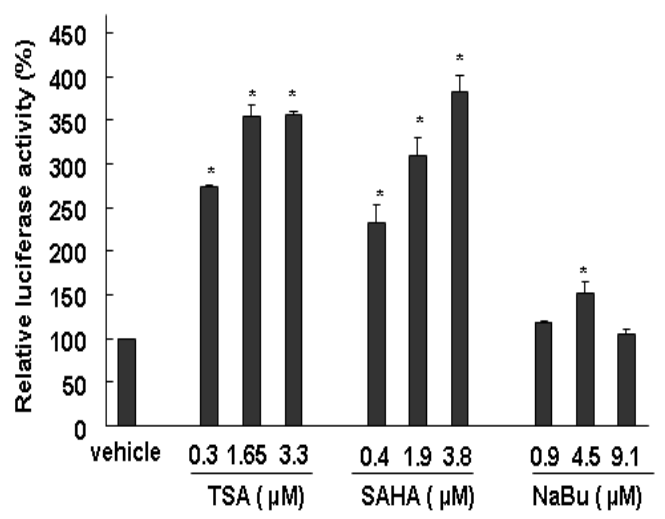

(C)

(A) The effect of 9179B on ABCA1 transcription in a cell-based model for ABCA1 up-regulators. The ABCA1p-LUC HepG2 cells were treated with serial dilutions of 9179B. The luciferase activity was measured $18 \mathrm{~h}$ later. The values represent the mean \pm S.D. of three separate experiments; $(\mathbf{B})$ HDAC inhibitory activity was measured at different concentrations of 9179B ( $1 \mathrm{nM}$ to $20 \mu \mathrm{M})$ with $10 \mu \mathrm{M}$ Fluor de $\mathrm{Lys}^{\mathrm{TM}}$ as the substrate. Values represent the mean \pm S.D. of three separate experiments; (C) The ABCA1p-LUC HepG2 cells were treated with different concentrations of TSA, SAHA and NaBu. The luciferase activity was measured $18 \mathrm{~h}$ later. Values represent the mean \pm S.D. of three separate experiments. The asterisk denotes a significant difference $(* p<0.05)$.

9179B is a derivative of trichostatin A (TSA), an inhibitor of histone deacetylase (HDAC) [19-21]. To test the inhibitory effect of 9179B on HDAC, the enzyme activities in the presence of different concentrations of $9179 \mathrm{~B}$ were measured. Figure $2 \mathrm{~B}$ shows that $9179 \mathrm{~B}$ inhibits HDAC activity in a dose-dependent manner, with an $\mathrm{IC}_{50}$ value of $0.08 \mu \mathrm{M}$. These results indicate that $9179 \mathrm{~B}$ is a potent HDAC inhibitor.

To investigate whether the up-regulation of ABCA1 promoter activity by $9179 \mathrm{~B}$ is due to its inhibition of HDAC, three other HDAC inhibitors, TSA, suberoylanilide hydroxamic acid (SAHA) and sodium butyrate $(\mathrm{NaBu})$, were used to compare their inhibition of ABCA1 transcription in ABCA1p-LUC HepG2 cells. These three inhibitors also increased the transcriptional activities of 
$\mathrm{ABCA} 1$ promoter, and reached at a maximum value of $355 \%$ at $3.3 \mu \mathrm{M}, 381 \%$ at $3.8 \mu \mathrm{M}$ and $152 \%$ at $4.5 \mu \mathrm{M}$, respectively (Figure $2 \mathrm{C}$ ).

The transcriptional regulation of ABCA1 is a highly complex process. Nuclear receptors involved in lipid homeostasis require the recruitment of coactivators and corepressors, collectively termed coregulators [22]. Coactivators are capable of recruiting the basal transcription factors and histone acetylases (HAT), which open the chromatin structure and induce transcription. Corepressors inhibit transcription via HDACs [23]. For example, the nuclear receptor corepressor (N-CoR), the silencing mediator of retinoic acid, and thyroid hormone receptors (SMRT) are essential components of the corepressor complex and they repress transcription by activating HDACs in the complex [24-26]. Therefore, this is the first report showing that 9179B is a HDAC inhibitor (HDACi) (Figure 2B) and can remarkably increase ABCA1 expression as well. We speculate that 9179B promotes ABCA1 transcription by modulating histone deacetylation in the promoter region of ABCA1 gene.

A HDAC inhibitor TSA was noticed to decrease the expression of SRA in P388D1 macrophage, suggesting that HDAC inhibition may prevent the development of AS [27]. Inflammation contributes to the formation and progression of AS and HDAC inhibitors have been shown to exhibit anti-inflammatory activities. Anti-inflammatory treatment regimens used in tumor necrosis factor- $\alpha$ blockage, IL-1 receptor antagonism, and leukotriene blockage may be beneficial for patients with coronary artery disease. Treatment of inflammation in atherosclerotic cardiovascular disease is thought to be emerging therapies $[28,29]$.

\subsection{The Effects of $9179 B$ on ABCA1 Expression in HepG2 and RAW 264.7 Cells and on Cholesterol Efflux in RAW 264.7 Cells}

To determine whether the induction of ABCA1 expression by 9179B was due to the up-regulation of transcription, quantitative real time RT-PCR was performed to investigate the abundance of ABCA1 mRNA and protein in HepG2 cells with and without 9179B treatment. Our data showed that 9179B increased the mRNA expression of ABCA1 in a dose-dependent manner in HepG2 cells, reaching about 5 folds at $17.5 \mu \mathrm{M}$ (Figure $3 \mathrm{~A}$ ).

Figure 3. The effects of 9179B on the expression of ABCA1 in HepG2 and RAW 264.7 cells and on cholesterol efflux in RAW 264.7 Cells.

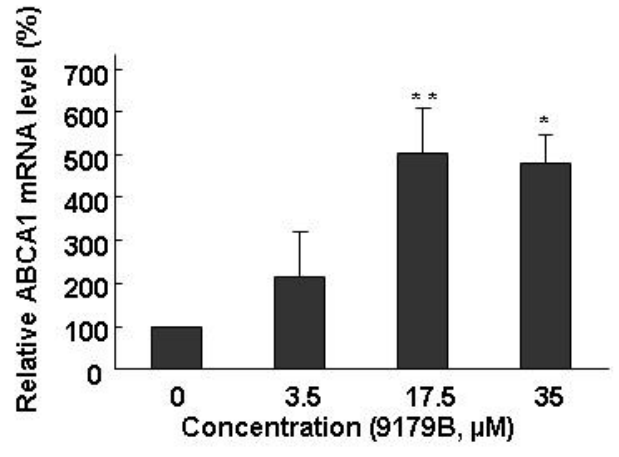

(A)

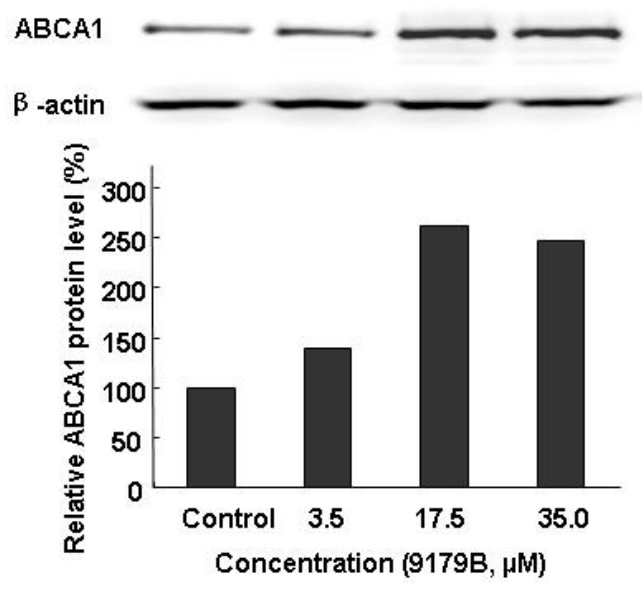

(B) 
Figure 3. Cont.

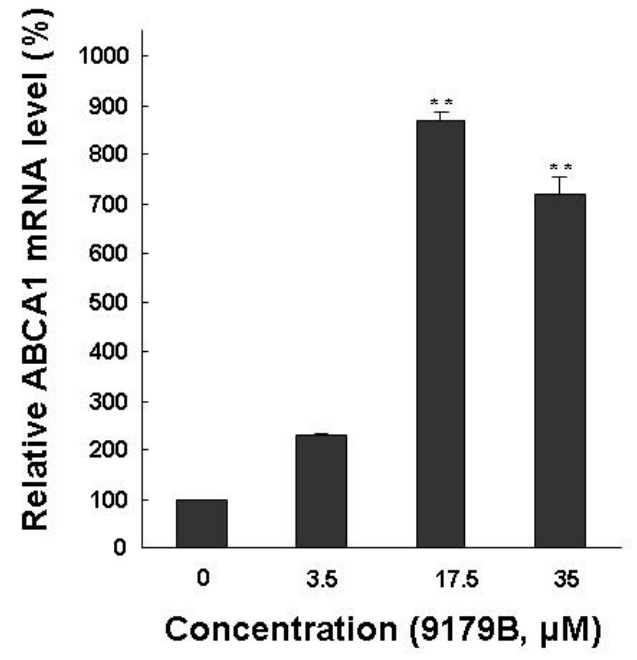

(C)

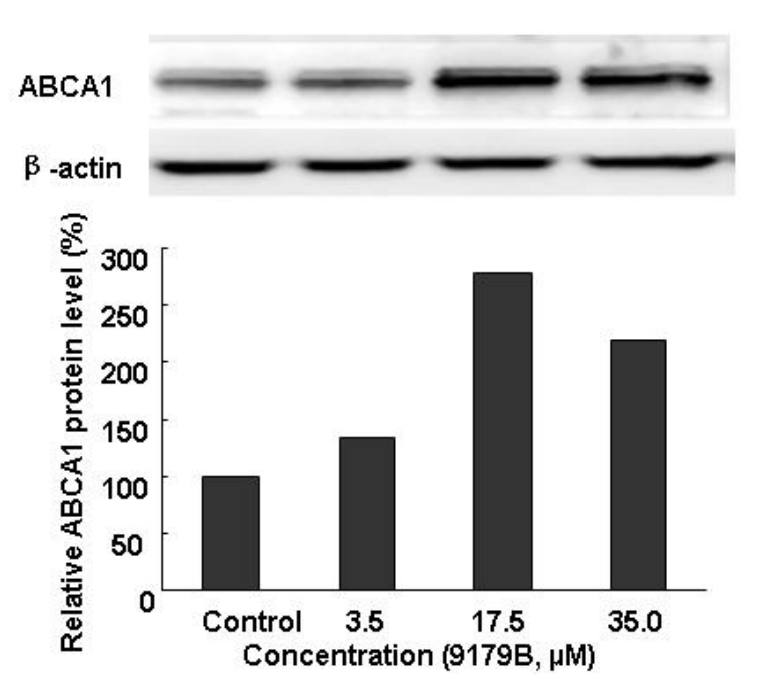

(D)

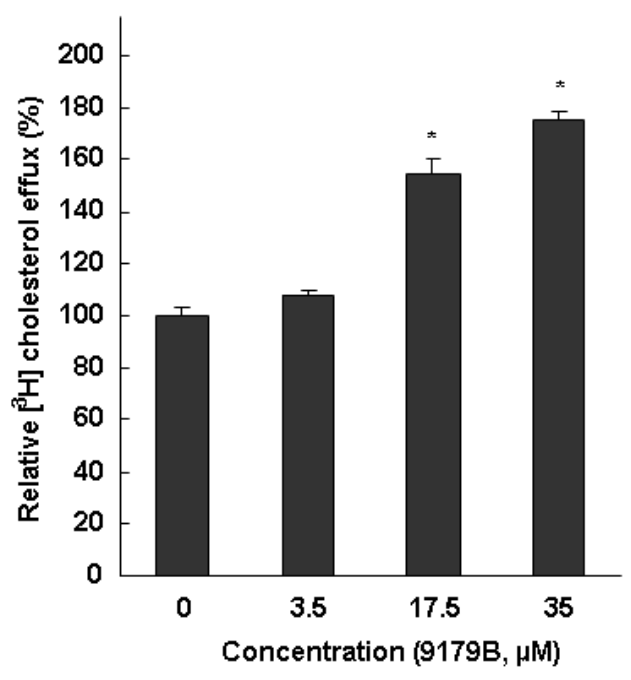

(E)

9179B stimulates ABCA1 transcription in HepG2 cells (A) and RAW264.7 cells (C). The ABCA1 mRNA level was detected using real time RT-PCR analysis. Values represent the mean \pm S.D. of three independent experiments. The asterisk denotes a significant difference $\left({ }^{*} p<0.05\right.$ and ** $p<0.01)$. 9179B increases the level of ABCA1 protein in HepG2 cells (B) and RAW264.7 cells (D). The cell surface ABCA1 protein level was detected using western blot analysis. A representative immunoblot is shown, demonstrating the abundance of the immunoreactive ABCA1 normalized to $\beta$-actin. 9179B stimulates ApoA-I-mediated cellular cholesterol efflux from RAW 264.7 cells $(\mathbf{E}) .\left[{ }^{3} \mathrm{H}\right]$ cholesterol-loaded macrophages were treated with $9179 \mathrm{~B}$ and subsequently incubated with DMEM medium with or without apoA-I $(15 \mu \mathrm{g} / \mathrm{mL})$ as indicated. ApoA-I-induced $\left[{ }^{3} \mathrm{H}\right]$ cholesterol efflux was measured as described. Values are the mean \pm S.D of three independent experiments, statistically significant differences are indicated $(* p<0.05)$.

In agreement, the protein expression of ABCA1 was also increased by 9179B treatment in a dose-dependent manner in HepG2 cells, reaching about 1.6-fold at 17.5 $\mu \mathrm{M}$ (Figure 3B). Cholesterol may enter macrophages via several different pathways and induce the transformation of macrophages into foam cells, the first step in atherosclerotic lesion development. ABCA1-dependent cholesterol efflux to lipid-poor apoA-I is a crucial factor in the prevention of excessive cholesterol accumulation 
in macrophages of the arterial wall and their transformation into foam cells [1]. Therefore, the effect of 9179B on ABCA1 expression in RAW264.7 cells was examined. Quantitative real time RT-PCR analysis indicated that 9179B increased the mRNA expression of ABCA1 in a dose-dependent manner in RAW 264.7 cells, reaching more than 8-fold higher at $17.5 \mu \mathrm{M}$ (Figure 3C). Consistently, the protein expression of ABCA1 was increased by 9179B treatment in a dose-dependent manner in RAW264.7 cells, reaching about 1.8 -fold at $17.5 \mu \mathrm{M}$ (Figure 3D).

Taken together, our data clearly demonstrate that the small molecule 9179B increases ABCA1 expression at both mRNA and protein levels in HepG2 and RAW 264.7 cells. Previous studies have suggested that ABCA1 is anti-atherogenic. Hepatocyte and macrophage ABCA1 plays important roles in HDL biosynthesis and cellular cholesterol efflux, respectively [4,30]. It is thus conceivable that 9179B as a novel and potent ABCA1 up-regulator may be beneficial for the prevention of atherosclerotic diseases.

In order to investigate the potential role of the active compound 9179B in modulating cholesterol efflux from mouse peritoneal macrophages, $\left[{ }^{3} \mathrm{H}\right]$ cholesterol efflux assay was performed using RAW264.7 cells. As shown in Figure 3E, treatment of RAW264.7 cells with 9179B resulted in a significant increase in cholesterol efflux to extracellular ApoA-I. The ratio of $\left[{ }^{3} \mathrm{H}\right]$ cholesterol efflux was $154.5 \%$ and $175.6 \%$ at $17.5 \mu \mathrm{M}$ and $35 \mu \mathrm{M}$ of $9179 \mathrm{~B}$, respectively.

Removal of cholesterol from macrophages via RCT is of significant importance in the prevention of atherosclerosis development. ABCA1 plays a pivotal role in RCT, and facilitates the efflux of cellular cholesterol to ApoA-I [31,32]. Drugs that induce ABCA1 expression in mice increased clearance of cholesterol from tissues [33]. 9179B could upregulate ABCA1 expression in HepG2 and RAW264.7 cells. It could be concluded that $\left[{ }^{3} \mathrm{H}\right]$ cholesterol efflux induced by $9179 \mathrm{~B}$ was the result of the upregulation of $\mathrm{ABCA} 1$ expression. This is a benefit to the prevention of atherosclerosis.

\subsection{The Influence of $9179 B$ on SR-BI and the Uptake of DiI-HDL in RAW 264.7 Cells}

IA04-9179 was also found to upregulate CLA-1 (human homologue of SR-BI) in a CLA-1-LUC cell line. In agreement with this finding, as a known compound trichostatin A (TSA), 9179A has been isolated and shown to upregulate CLA-1/SR-BI expression [34]. Similar effects on the expression of SR-BI were observed for 9179B. RAW264.7 cells were used to examine the effect of 9179B on SR-BI. After treatment with $3.5 \mu \mathrm{M}, 17.5 \mu \mathrm{M}$ and $35.0 \mu \mathrm{M}$ 9179B, Western blot assay showed that 9179B could up-regulate SR-BI expression in a dose-dependent manner (Figure 4A). At $35.0 \mu \mathrm{M} 9179 \mathrm{~B}$ could significantly increase the SR-BI protein levels by $97.1 \%$.

SR-BI is the main receptor of HDL and it is expressed in macrophages and plays an important role in the process of reverse cholesterol transport (RCT) [21]. Since 9179B increases the expression level of SR-BI, we tested whether 9179B could enhance the uptake of HDL. The uptake of fluorescently labeled DiI-HDL was measured after $4 \mathrm{~h}$ incubation in the presence of different concentrations of 9179B or vehicle $(0.1 \%$ DMSO) in RAW 264.7 cells. The results showed that $3.5 \mu \mathrm{M}$ and $17.5 \mu \mathrm{M}$ 9179B promotes DiI-HDL uptake into RAW 264.7 cells by $29.5 \%$ and $153 \%$, respectively (Figure 4B). 
Figure 4. The effect of 9179B on the expression of SR-BI in RAW 264.7 cells and flow cytometry analysis of the uptake of DiI-HDL by RAW 264.7 cells.

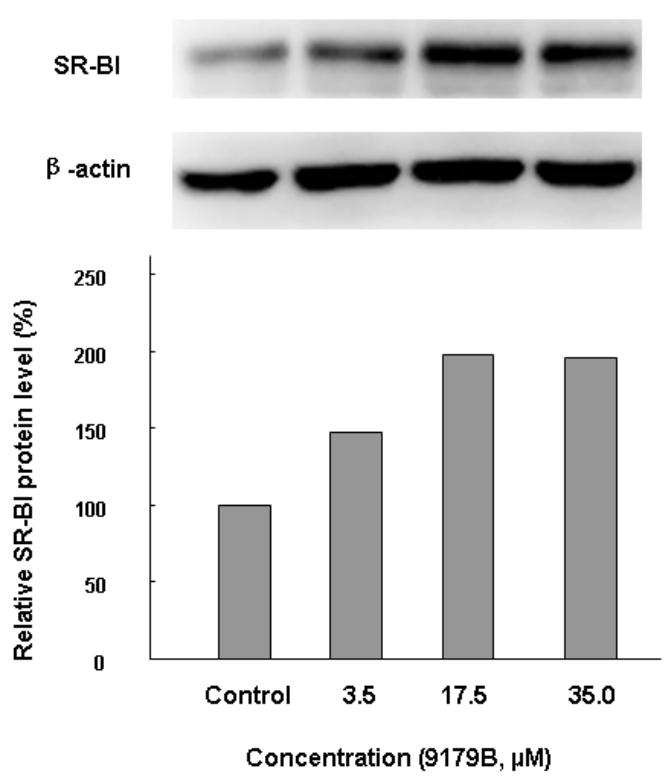

(A)
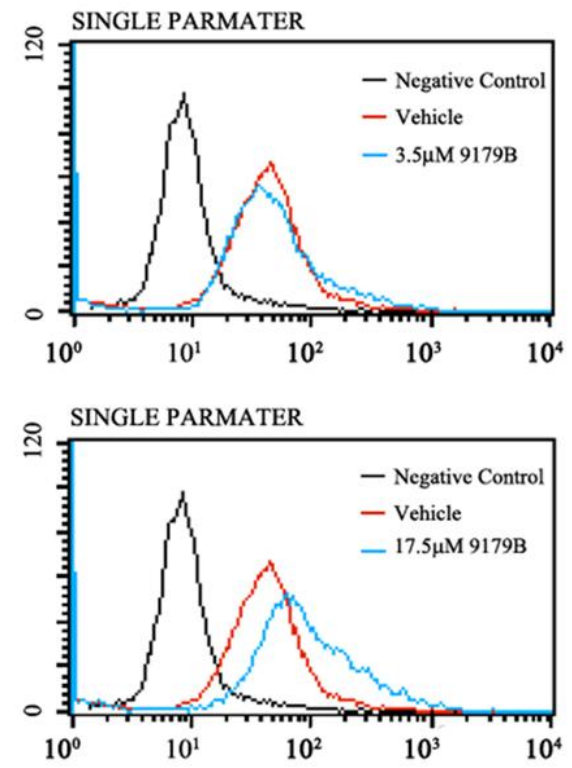

(B)

RAW 264.7 cells were treated with 9179B at the indicated concentrations, and SR-BI expression (A) and the uptake of DiI-HDL (B) was measured as described in the Experimental. Western blot analysis showed that 9179B increased the protein level of SR-BI (A). A representative immunoblot is shown to demonstrate the abundance of the immunoreactive SR-BI protein normalized to $\beta$-actin. The uptake of DiI-HDL (B) was measured by flow cytometry. Cells for negative control were neither treated with 9179B nor incubated with DiI-HDL. Vehicle cells were not treated with 9179B before incubation with $2 \mu \mathrm{g} / \mathrm{mL}$ of DiI-HDL.

9179B increases the uptake of DiI-HDL in RAW264.7 cells, which may be caused by the up-regulation of SR-BI expression by 9179B. The increased uptake of DiI-HDL might result in cholesterol efflux from macrophages. Taken together, our results suggest that 9179B may facilitate the efflux of cellular cholesterol to extracellular acceptors through the induction of ABCA1 and SR-BI expression and/or their activities, which will benefit to AS.

\subsection{The Influence of $9179 B$ on Different Cis-Regulatory Elements of ABCA1}

To identify the putative response domain in the ABCA1 promoter, seven different promoter-fragment/luciferase reporter constructs with progressive 5' end deletions were transiently expressed in HepG2 cells. The basal promoter activity decreased significantly upon removal of the base pairs between -719 and -582 . Removal of the base pair from -171 to -78 further reduced the promoter activity (Figure 5A). We tested whether 9179B could induce transcription with these fragments of ABCA1 promoter. Figure 5B shows that the transcriptional activity between $-171 /+67 \mathrm{bp}$ and $-78 /+67 \mathrm{bp}$ fragments was significantly different. 
Figure 5. Characterization of the DNA motifs mediating 9179B up-regulation of ABCA1 promoter activity.

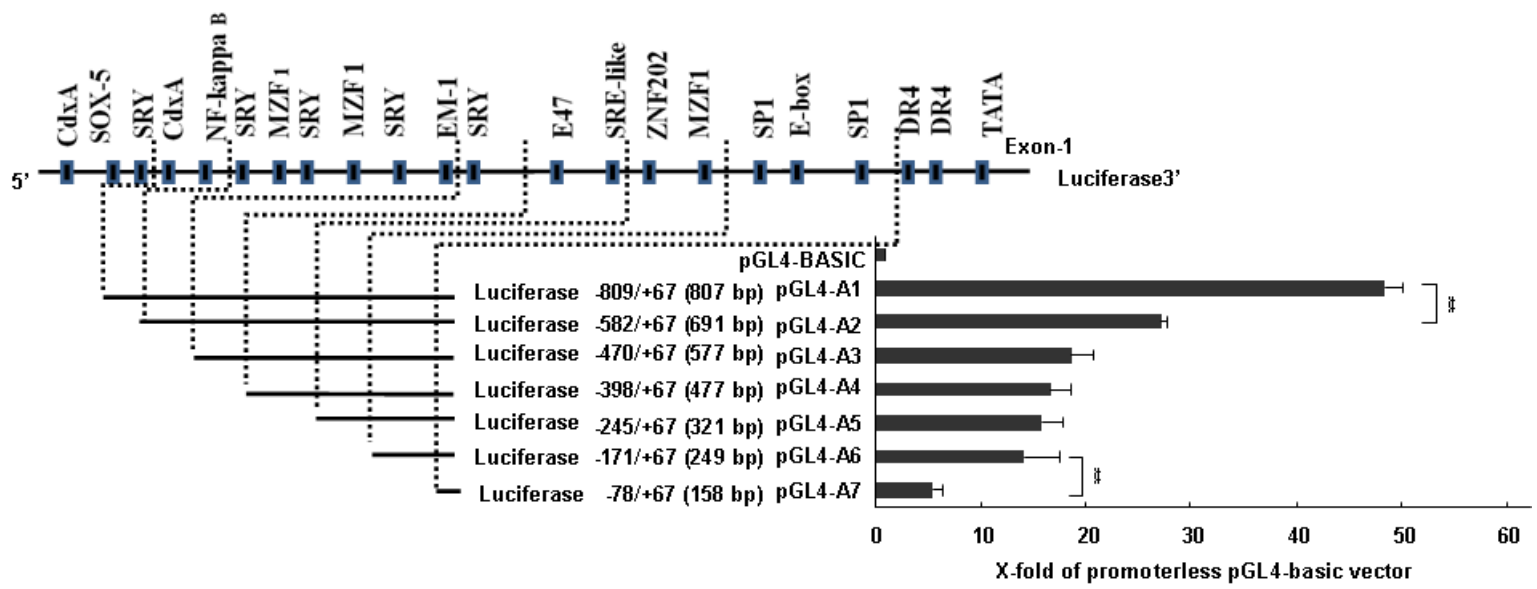

(A)

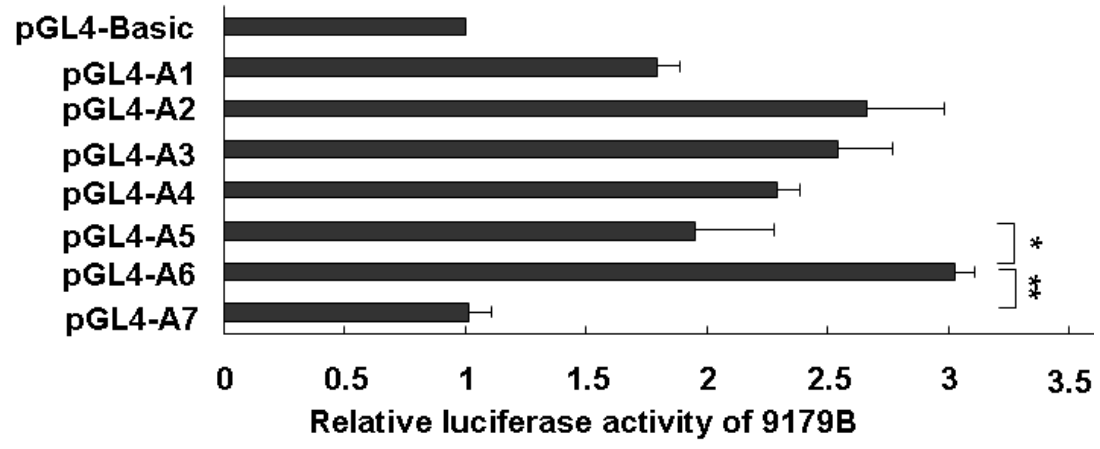

(B)

(A) The schematic of ABCA1 promoter region containing functional cis-regulatory elements and the relative luciferase activity of different constructs in transiently transfected HepG2 cells; (B) Deletion analysis of the ABCA1 promoter mapped the 9179B responsive region. HepG2 cells were transiently transfected with different constructs and treated with $17.5 \mu \mathrm{M} 9179 \mathrm{~B}$. The average of five separate experiments performed in duplicate is shown as the mean \pm S.D. The asterisks denote a significant difference $(* p<0.05$ and $* * p<0.01)$.

When the $-171 /-78$ bp fragment was removed, the luciferase activity decreased dramatically. This indicates that the $-171 /-78 \mathrm{bp}$ fragment is important for $9179 \mathrm{~B}$ to induce ABCA1 transcription. In this study, the cis-element of the ABCA1 promoter responsible for the 9179B up-regulation was mapped to the $-171 /-78$ bp fragment (Figure 5B). Previous studies showed that two SP1 sites (the -91 and -157 GnC motifs) and an E-box (-140) motif were identified within the $-171 /-75$ bp region [35,36]. SP1 plays a critical role in the formation or recruitment of the transcription initiation complex at the core promoter and also regulates the expression of proteins and enzymes involved in lipoprotein metabolism [35,36]. Overexpression of SP1 increased ABCA1 mRNA expression in HeLa cells. The E-box motif mediates transcriptional repression of the ABCA1 gene via basic helix-loop-helix transcription factors [35,37]. Removing the $-171 /-78$ bp fragment, the luciferase activity decreased significantly. Therefore, we reason that the cis-element SP1 contributes to the up-regulation of the ABCA1 transcription by $9179 \mathrm{~B}$. These results suggest that 9179B may activate ABCA1 gene 
transcription by inhibiting deacetylase activity, or by activating the SP1 cis-element or through both mechanisms.

\section{Experimental}

\subsection{General}

The HDAC Fluorimetric Assay/Drug Discovery Kit was obtained from Enzo Life Science (Plymouth Meeting, PA, USA). The rabbit polyclonal antibody for ABCA1 was purchased from Novus Biologicals (Littleton, CO, USA). 1,1'-Dioctadecyl-3,3,3',3'-tetramethylindocarbocyanine perchlorate-labeled HDL (DiI-HDL) was purchased from Biomedical Technologies (Stoughton, MA, USA). White-wall, clear bottom 96-well polystyrene assay plates were obtained from BMG LABTECH (Offenburg, Germany). 60-mm dishes and 24- and 96-well plates for cell culture were obtained from Corning (Acton, MA, USA). Cell culture media and fetal bovine serum (FBS) were bought from Hyclone (Logan, UT, USA). G418 was obtained from Invitrogen (Carlsbad, CA, USA).

\subsection{Cell Culture}

HepG2 human hepatoma cells were grown in minimum essential medium with Earle's balanced salts and $2 \mathrm{mM}$ L-glutamine solution (MEM-EBSS) containing 10\% FBS (medium A). ABCA1p-LUC HepG2 cells, which are HepG2 cells stably transfected with a gene construct in which the promoter and regulatory control elements for the human ABCA1 gene were fused to a luciferase reporter gene. The transfected cells were grown in medium A supplemented with $600 \mu \mathrm{g} / \mathrm{mL}$ G418 (medium B). RAW264.7 murine macrophage cells were grown in Dulbecco's modified Eagle's medium (DMEM, high glucose) containing 10\% FBS (medium C). All cells were maintained in a humidified $5 \% \mathrm{CO}_{2}$ incubator at $37^{\circ} \mathrm{C}$.

\subsection{The Isolation, Purification and Structure Elucidation of $9179 B$}

The fermentation of strain 04-9179 was performed as previously described [31]. The fermentation filtrate ( 4 30 liters) was applied to a HP-20 macroporous resin column (Mitsubishi Chemical, Tokyo, Japan), which was washed with water and eluted with aqueous acetone $(30 \%, 50 \%, 80 \%, 100 \%)$. The $50 \%$ acetone eluting fractions with enhanced luciferase activity were collected and concentrated in vacuo. The dry residue was dissolved in methanol and loaded on a $4 \times 300 \mathrm{~cm}$ column of octadecyltrichlorosilane (ODS; YMC, Kyoto, Japan). The column was eluted with a three-column bed volume of aqueous methanol $(10 \%, 20 \%, 30 \%, 40 \%, 45 \%, 50 \%, 55 \%, 60 \%, 80 \%, 100 \%)$. The eluates were analyzed by high performance liquid chromatography (ODS column, $150 \times 4.6 \mathrm{~mm}, 5 \mu \mathrm{m}$, Shimadzu, Kyoto, Japan) with $\mathrm{MeOH}$-water (50:50) as eluent. The fractions that can upregulate ABCA1 transcription were evaporated and concentrated in vacuo. Final purification was achieved using semipreparative HPLC $(250 \times 9.4 \mathrm{~mm}, 5 \mu \mathrm{m}$, Agilent, CA, USA), which was eluted with $52 \%$ $\mathrm{MeOH}$-water. The pure active compound, 9179B, was obtained and concentrated to dryness. The physicochemical properties of 9179B were determined via UV spectrometry (UV-200S, Shimadzu, Kyoto, Japan), mass spectrometry (JMS-DX 300, Jeol, Tokyo, Japan), and NMR spectrometry 
(XL-400, Varian, Palo Alto, CA, USA). The structure of 9179B was elucidated by spectral data from

UV, ESI, FAB, ${ }^{1} \mathrm{H}-\mathrm{NMR},{ }^{13} \mathrm{C}-\mathrm{NMR}$, HSQC, HMBC, and DEPT with $\mathrm{CD}_{3} \mathrm{OD}$ as the solvents.

\subsection{ABCA1 Promoter Activity Assay}

The ABCA1 transcriptional activity assay was carried our as described previously [18]. Briefly, the ABCA1p-LUC HepG2 cells were plated in 96-well dishes at 500,000 cells/well in $100 \mu \mathrm{L}$ medium B for $12 \mathrm{~h}$. Then the cells were treated with the indicated concentrations of $9179 \mathrm{~B}$ or vehicle $(0.1 \%$ DMSO). After being incubated for $18 \mathrm{~h}$ at $37^{\circ} \mathrm{C}$, cells were washed with PBS (137 mM NaCl, $2.7 \mathrm{mM}$ $\mathrm{KCl}, 4.3 \mathrm{mM} \mathrm{Na}_{2} \mathrm{HPO}_{4}, 1.4 \mathrm{mM} \mathrm{KH} \mathrm{PO}_{4}, \mathrm{pH} 7.3$ ), and then the luciferase activity was detected as relative luminescence units (RLUs) using the Luciferase Assay System (Promega, Madison, WI, USA).

\subsection{Enzyme Inhibitor Activity Assay and Other HDAC Inhibitor on ABCA1 Promoter Activity}

HDAC inhibition activity was assayed using the Fluorimetric Assay/Drug Discovery Kit (BML-AK500) according to the manufacturer's protocol. The fluorescence intensity was measured using a fluorescence microplate reader (EnVision Multilabel Reader, PerkinElmer, Waltham, MA, USA) (excitation $360 \mathrm{~nm}$, emission $460 \mathrm{~nm}$ ). The ability of 9179B to inhibit HDAC activity was measured at the indicated concentrations of $9179 \mathrm{~B}$ with $10 \mu \mathrm{M}$ Fluor de Lys as substrate. The $\mathrm{IC}_{50}$ was calculated using Origin 7.5 software. The ABCA1 transcriptional activity assay was performed as described in 2.2. The ABCA1p-LUC HepG2 cells were treated with the indicated concentrations of TSA, SAHA, NaBu or vehicle (0.1\% DMSO). After incubation for $18 \mathrm{~h}$ at $37^{\circ} \mathrm{C}$, cells were washed with PBS, and then the luciferase activity was detected as relative luminescence units (RLUs) using the Luciferase Assay System (Promega, Madison, WI, USA).

\subsection{The Effects of $9179 B$ on ABCA1 Expression in HepG2 or RAW264.7 Cells}

HepG2 or RAW264.7 cells were incubated with 9179B at the indicated concentrations for $24 \mathrm{~h}$ and then were washed twice with PBS. Total cellular RNA and protein extracts were obtained as described before [18].

For quantitative real time PCR, SYBR Green PCR Core Reagent (Applied Biosystems, Foster City, CA, USA) was used on an iQ5 Multicolor Real-Time PCR Detection System (Bio-Rad, Hercules, CA, USA). The following sequences of primers (sense and antisense) were used for amplification: 5'-GCCTGCTAGTGGTCATCCTG-3' and 5'-CCACGCTGGGATCACTGTA-3' (for human ABCA1), 5'-TCCACTGCGTCTTCACC-3' and 5'-GGCAGAGATGATGACCCTTTT-3' (for human GAPDH); 5'-GGGTCTGAACTGCCCTACCT-3' and 5'-TACTCCCCTGATGCCACTTC-3' (for mouse ABCA1), 5'-CTAAGGCCAACCGTGAAAG-3' and 5'-ACCAGAGGCATACAGGGACA-3' (for mouse $\beta$-actin). Expression data were normalized for GAPDH and $\beta$-actin levels.

For Western blotting, protein extracts $(25 \mu \mathrm{g})$ from control or treated cells were subjected to $10 \%$ SDS-polyacrylamide gel electrophoresis. Then proteins were transferred to a $0.45-\mu \mathrm{m}$ polyvinylidene difluoride (PVDF) membrane (Millipore, Bedford, MA, USA). The membrane was blocked for $1 \mathrm{~h}$ at room temperature in TBS contain $0.1 \%$ Tween-20 (TBST) in the presence of 5\% nonfat dry milk. Membranes were incubated with the rabbit polyclonal antibody for ABCA1 protein (1:500 dilution, 
$4{ }^{\circ} \mathrm{C}$, overnight) (Novus Biologicals, Littleton, CO, USA) in TBST containing 5\% nonfat dry milk. Then membranes were washed by TBST and incubated with horseradish peroxidase-conjugated goat antirabbit IgG (Novus Biologicals) in TBST containing 5\% nonfat dry milk for $1 \mathrm{~h}$ at room temperature (1:2000 dilution). An enhanced chemiluminiscence detection system (Santa Cruz Biotechnology, Santa Cruz, CA, USA) was used for detection. Human $\beta$-actin was simultaneously detected as an internal control.

\subsection{The Effects of $9179 B$ on Cholesterol Efflux in RAW 264.7 Cells}

The cholesterol efflux assay was done as previously described [38]. RAW264.7 cells were plated in 96-well plates and incubated for $24 \mathrm{~h}$. Then they were incubated with DMEM containing $2 \mu \mathrm{Ci}$ of $\left[1,2-{ }^{3} \mathrm{H}\right]$ cholesterol $(1 \mathrm{mCi} / \mathrm{mL}$; PerkinElmer, Waltham, MA,USA) per $\mathrm{mL}$ for $24 \mathrm{~h}$. Then the cells were washed three times with PBS containing 0.1\% BSA (PBS-BSA), and fresh DMEM containing $0.1 \%$ BSA (DMEM-BSA) with $9179 \mathrm{~B}$ at the indicated concentrations added for $24 \mathrm{~h}$. After intensive washing of cells with PBS-BSA, ApoA-I $(15 \mu \mathrm{g} / \mathrm{mL})$ prepared in DMEM-BSA as the cholesterol acceptor were added and incubated for $24 \mathrm{~h}$. Finally, medium was collected and centrifuged $(10,000 \times \mathrm{g}$ for $5 \mathrm{~min})$, and the radioactivity was counted by liquid scintillation counting. The residual radioactivity in the cell fraction was determined after half hour hydrolysis with $0.1 \mathrm{M} \mathrm{NaOH}$. The ratio of $\left[{ }^{3} \mathrm{H}\right]$ efflux was calculated by dividing the radioactive counts in the medium by the sum of the radioactive counts in the medium and the cell fraction. DMEM-BSA was used as the blank, and the efflux of vehicle treated cells was expressed as $100 \%$.

\subsection{The Effect of 9179B on SR-BI Expression in RAW264.7 Cells and the Analysis of Cellular Uptake of DiI-Labeled HDL by Flow Cytometry}

RAW264.7 cells were incubated with 9179B at the indicated concentrations for $24 \mathrm{~h}$ and then were washed twice with PBS. For Western blots, protein extracts $(25 \mu \mathrm{g})$ from control or treated cells were subjected to $10 \%$ SDS-polyacrylamide gel electrophoresis. Then proteins were transferred to a 0.45- $\mu \mathrm{m}$ polyvinylidene difluoride (PVDF) membrane (Millipore, Bedford, MA, USA). The membrane was blocked for $1 \mathrm{~h}$ at room temperature in TBS contain $0.1 \%$ Tween-20 (TBST) in the presence of $5 \%$ nonfat dry milk. Membranes were incubated with the rabbit polyclonal antibody SR-BI (1:1,000 dilution, $4{ }^{\circ} \mathrm{C}$, overnight) (Novus Biologicals, Littleton, CO, USA) in TBST containing 5\% nonfat dry milk. Then membranes were washed by TBST and incubated with horseradish peroxidase-conjugated goat antirabbit IgG (Novus Biologicals) in TBST containing 5\% nonfat dry milk for $1 \mathrm{~h}$ at room temperature (1:3000 dilution). An enhanced chemiluminiscence detection system (Santa Cruz Biotechnology, Santa Cruz, CA, USA) was used for detection. Mouse $\beta$-actin was simultaneously detected as an internal control to monitor the intensity of the immunoreactive SR-BI.

RAW264.7 cells were plated at 50,000 cells/well in 24-well dishes. The cells were treated for $18 \mathrm{~h}$ with 9179B at specified concentrations. Then DiI-HDL (final concentration $2.0 \mu \mathrm{g} / \mathrm{mL}$ ) was added at $37{ }^{\circ} \mathrm{C}$ for $4 \mathrm{~h}$. The cells were washed twice with cold PBS and incubated in PBS supplemented with $0.5 \%$ bovine serum albumin and $2 \mathrm{mM}$ EDTA at $4{ }^{\circ} \mathrm{C}$ for $1 \mathrm{~h}$. The cell suspensions were transferred and centrifuged ( $3 \mathrm{~min}, 800 \mathrm{~g}, 4{ }^{\circ} \mathrm{C}$ ) and then were resuspended in PBS. DiI fluorescence was analyzed by an Epics XL flow cytometer (Coulter Corporation, Miami, FL, USA). 


\subsection{Luciferase Reporter Assay}

The reporter constructs contained the ABCA1 upstream regulatory sequence region from -719 to $-67 \mathrm{bp}$ (nucleotide +1 corresponds to the A of the ATG start codon). pGL4-ABCA1-1 (-719/+67, 807 bp), pGL4-ABCA1-2 (-582/+67, 691 bp), pGL4-ABCA1-3 (-470/+67, 577 bp), pGL4-ABCA1-4 (-398/+67, 477 bp), pGL4-ABCA1-5 (-245/+67, 321 bp), pGL4-ABCA1-6 (-171/+67, 249 bp) and pGL4-ABCA1-7 (-78/+67, 158 bp) were purified using PureYield ${ }^{\mathrm{TM}}$ Plasmid Midiprep System (Promega). The reporter plasmid $(0.2 \mu \mathrm{g})$ was transfected into 50,000 HepG2 cells using the cationic liposome transfection method (Lipofectamine 2000, Invitrogen). After reporter plasmids were transfected for $5 \mathrm{~h}$, then HepG2 cells were incubated with $17.5 \mu \mathrm{M} 9179 \mathrm{~B}$ or vehicle $(0.1 \% \mathrm{DMSO})$ in MEM medium without FBS. Cells were incubated for $18 \mathrm{~h}$ and the luciferase activity was detected as RLUs by the Luciferase Assay System (Promega).

\subsection{Statistical Analysis}

All experimental results were expressed as means \pm S.D. Statistical significance of the data was evaluated by the Student's $t$-test. A significant difference was indicated at $p<0.05$.

\section{Conclusions}

In this paper we report for the first time the identification and characterization of dehydroxytrichostatin A (9179B) as a novel up-regulator of ABCA1. Dehydroxytrichostatin A upregulates ABCA1 expression at the mRNA and protein levels in HepG2 cells and RAW264.7 cells, and increases DiI-HDL uptake in RAW264.7 cells. 9179B stimulated ApoA-I-mediated cellular cholesterol efflux from RAW 264.7 cells. Moreover, we found that 9179B was a potent HDAC inhibitor. Our results also suggest that 9179B may regulate ABCA1 transcription by affecting the interaction of HDAC or SP1 with the ABCA1 promoter. These results show the significance of further studies of ABCA1 transcriptional regulation. In conclusion, our studies have demonstrated that 9179B is a novel up-regulator of ABCA1, which could be used as an anti-atherogenic drug.

\section{Acknowledgments}

This work was supported by the State Key New Drug Creation and Manufacturing Program (2009ZX09302-004, 2009ZX09301-003 and 2008ZX09401-005) and a grant from the National Natural Science Foundation of China (grant number 90813027).

\section{References}

1. Ross, R. Atherosclerosis-An inflammatory disease. N. Engl. J. Med. 1999, 340, 115-126.

2. Stein, O.; Stein, Y. Atheroprotective mechanisms of HDL. Atherosclerosis 1999, 144, 285-301.

3. Gordon, D.J.; Probstfield, J.L.; Garrison, R.J.; Neaton, J.D.; Castelli, W.P.; Knoke, J.D.; Jacobs, D.R., Jr.; Bangdiwala, S.; Tyroler, H.A. High-density lipoprotein cholesterol and cardiovascular disease. Four prospective American studies. Circulation 1989, 79, 8-15. 
4. Goldbourt, U.; Yaari, J.S.; Medalie, J.H. Isolated low HDL cholesterol as a risk factor for coronary heart disease mortality: A 21-year follow-up of 8,000 men. Arterioscler. Thromb. Vasc. Biol. 1997, 17, 107-113.

5. Langmann, T.; Klucken, J.; Reil, M.; Liebisch, G.; Luciani, M.F.; Chimini, G.; Kaminski, W.E.; Schmitz, G. Molecular cloning of the human ATP-binding cassette transporter 1 (hABC1): Evidence for sterol-dependent regulation in macrophages. Biochem. Biophys. Res. Commun. 1999, 257, 29-33.

6. Lee, J.Y.; Parks, J.S. ATP-binding cassette transporter AI and its role in HDL formation. Curr. Opin. Lipidol. 2005, 16, 19-25.

7. Oram, J.F.; Vaughan, A.M. ABCA1-mediated transport of cellular cholesterol and phospholipids to HDL apolipoproteins. Curr. Opin. Lipidol. 2000, 11, 253-260.

8. Zannis, V.I.; Chroni, A.; Krieger, M. Role of apoA-I, ABCA1, LCAT, and SR-BI in the biogenesis of HDL. J. Mol. Med. 2006, 84, 276-294.

9. Zhao, C.; Dahlman-Wright, K. Liver X receptor in cholesterol metabolism. J. Endocrinol. 2010, 204, 233-240.

10. Brooks-Wilson, A.; Marcil, M.; Clee, S.M.; Zhang, L.H.; Roomp, K.; van Dam, M.; Yu, L.; Brewer, C.; Collins, J.A.; Molhuizen, H.O.; et al. Mutations in ABC1 in Tangier disease and familial highdensity lipoprotein deficiency. Nat. Genet. 1999, 22, 336-345.

11. Bodzioch, M.; Orsó, E.; Klucken, J.; Langmann, T.; Böttcher, A.; Diederich, W.; Drobnik, W.; Barlage, S.; Büchler, C.; Porsch-Ozcürümez, M.; et al. The gene encoding ATP-binding cassette transporter 1 is mutated in Tangier disease. Nat. Genet. 1999, 22, 347-351.

12. Lu, L.; Liu, H.; Peng, J.; Gan, L.; Shen, L.; Zhang, Q.; Li, L.; Zhang, L.; Su, C.; Jiang, Y. Regulations of the key mediators in inflammation and atherosclerosis by aspirin in human macrophages. Lipids Health Dis. 2010, 9, 16.

13. Singaraja, R.R.; Fievet, C.; Castro, G.; James, E.R.; Hennuyer, N.; Clee, S.M.; Bissada, N.; Choy, J.C.; Fruchart, J.C.; McManus, B.M.; et al. Increased ABCA1 activity protects against atherosclerosis. J. Clin. Invest. 2002, 110, 35-42.

14. Joyce, C.W.; Amar, M.J.; Lambert, G.; Vaisman, B.L.; Paigen, B.; Najib-Fruchart, J.; Hoyt, R.F., Jr.; Neufeld, E.D.; Remaley, A.T.; Fredrickson, D.S.; et al. The ATP binding cassette transporter A1 (ABCA1) modulates the development of aortic atherosclerosis in C57BL/6 and apoE-knockout mice. Proc. Natl. Acad. Sci. USA 2002, 99, 407-412.

15. Timmins, J.M.; Lee, J.Y.; Boudyguina, E.; Kluckman, K.D.; Brunham, L.R.; Mulya, A.; Gebre, A.K.; Coutinho, J.M.; Colvin, P.L.; Smith, T.L.; et al. Targeted inactivation of hepatic Abcal causes profound hypoalphalipoproteinemia and kidney hypercatabolism of apoA-I. J. Clin. Invest. 2005, 115, 1333-1342.

16. Brunham, L.R.; Kruit, J.K.; Iqbal, J.; Fievet, C.; Timmins, J.M.; Pape, T.D.; Coburn, B.A.; Bissada, N.; Staels, B.; Groen, A.K.; et al. Intestinal ABCA1 directly contributes to HDL biogenesis in vivo. J. Clin. Invest. 2006, 116, 1052-1062.

17. Basso, F.; Freeman, L.; Knapper, C.L.; Remaley, A.; Stonik, J.; Neufeld, E.B.; Tansey, T.; Amar, M.J.; Fruchart-Najib, J.; Duverger, N.; et al. Role of the hepatic ABCA1 transporter in modulating intrahepatic cholesterol and plasma HDL cholesterol concentrations. J. Lipid Res. 2003, 44, 296-302. 
18. Gao, J.; Xu, Y.; Yang, Y.; Yang, Y.; Zheng, Z.; Jiang, W.; Hong, B.; Yan, X.; Si, S. Identification of upregulators of human ATP-binding cassette transporter A1 via high-throughput screening of a synthetic and natural compound library. J. Biomol. Screen. 2008, 13, 648-656.

19. Tsuji, N.; Kobayashi, M.; Nagashima, K.; Wakisaka, Y.; Koizumi, K. A new antifungal antibiotic, trichostatin. J. Antibiot. (Tokyo) 1976, 29, 1-6.

20. Tsuji, N.; Kobayashi, M. Trichostatin C, a glucopyranosyl hydroxamate. J. Antibiot (Tokyo). 1978, 31, 939-944.

21. Yoshida, M.; Nomura, S.; Beppu, T. Effects of trichostatins on differentiation of murine erythroleukemia cells. Cancer Res. 1987, 47, 3688-3691.

22. Rosenfeld, M.G.; Glass, C.K. Coregulator codes of transcriptional regulation by nuclear receptors. J. Biol. Chem. 2001, 276, 36865-36868.

23. Hermanson, O.; Glass, C.K.; Rosenfeld, M.G. Nuclear receptor coregulators: Multiple modes of modification. Trends Endocrinol. Metab. 2002, 13, 55-60.

24. Huang, E.Y.; Zhang, J.; Miska, E.A.; Guenther, M.G.; Kouzarides, T.; Lazar, M.A. Nuclear receptor corepressors partner with class II histone deacetylases in a Sin3-independent repression pathway. Genes Dev. 2000, 14, 45-54.

25. Guenther, M.G.; Lane, W.S.; Fischle, W.; Verdin, E.; Lazar, M.A.; Shiekhattar, R. A core SMRT corepressor complex containing HDAC3 and TBL1, a WD40-repeat protein linked to deafness. Genes Dev. 2000, 14, 1048-1057.

26. Wen, Y.D.; Perissi, V.; Staszewski, L.M.; Yang, W.M.; Krones, A.; Glass, C.K.; Rosenfeld, M.G.; Seto, E. The histone deacetylase-3 complex contains nuclear receptor corepressors. Proc. Natl. Acad. Sci. USA 2000, 97, 7202-7207.

27. Laribee, R.N.; Klemsz, M.J. Loss of PU.1 expression following inhibition of histone deacetylases. J. Immunol. 2001, 167, 5160-5166.

28. Klingenberg, R.; Hansson, G.K. Treating inflammation in atherosclerotic cardiovascular disease: Emerging therapies. Eur. Heart J. 2009, 30, 2838-2844.

29. Moubayed, S.P.; Heinonen, T.M.; Tardif, J.C. Anti-inflammatory drugs and atherosclerosis. Curr. Opin. Lipidol. 2007, 18, 638-644.

30. Schmitz, G.; Langmann, T. Transcriptional regulatory networks in lipid metabolism control ABCA1 expression. Biochim. Biophys. Acta 2005, 1735, 1-19.

31. Ohashi, R.; Mu, H.; Wang, X.; Yao, Q.; Chen, C. Reverse cholesterol transport and cholesterol efflux in atherosclerosis. QJM 2005, 98, 845-856.

32. Francis, G.A.; Knopp, R.H.; Oram, J.F. Defective removal of cellular cholesterol and phospholipids by apolipoprotein A-I in Tangier Disease. J. Clin. Invest. 1995, 96, 78-87.

33. Oram, J.F.; Lawn, R.M. ABCA1. The gatekeeper for eliminating excess tissue cholesterol. J. Lipid Res. 2001, 42, 1173-1179.

34. Bao, Y.; Yang, Y.; Wang, L.; Gao, L.; Jiang, W.; Wang, L.; Si, S.; Hong, B. Identification of trichostatin A as a novel transcriptional up-regulator of scavenger receptor BI both in HepG2 and RAW264.7 cells. Atherosclerosis 2009, 204, 127-135.

35. Langmann, T.; Porsch-Ozcürümez, M.; Heimerl, S.; Probst, M.; Moehle C.; Taher, M.; Borsukova, H.; Kielar, D.; Kaminski, W.E.; Dittrich-Wengenroth, E.; et al. Identification of sterol-independent regulatory elements in the human ATP-binding cassette transporter A1 
promoter: Role of Sp1/3, E-box binding factors, and an oncostatin M-responsive element. J. Biol. Chem. 2002, 277, 14443-14450.

36. Langmann, T.; Buechler, C.; Ries, S.; Schaeffler, A.; Aslanidis, C.; Schuierer, M.; Weiler, M.; Sandhoff, K.; de Jong, P.J.; Schmitz, G. Transcription factors Sp1 and AP-2 mediate induction of acid sphingomyelinase during monocytic differentiation. J. Lipid Res. 1999, 40, 870-880.

37. Yang, X.P.; Freeman, L.A.; Knapper, C.L.; Amar, M.J.; Remaley, A.; Brewer, H.B., Jr.; Santamarina-Fojo, S. The E-box motif in the proximal ABCA1 promoter mediates transcriptional repression of the ABCA1 gene. J. Lipid Res. 2002, 43, 297-306.

38. Baranova, I.; Vishnyakova, T.; Bocharov, A.; Chen, Z.; Remaley, A.T.; Stonik, J.; Eggerman, T.L.; Patterson, A.P. Lpopolysaccharide down regulates both scavenger receptor B1 and ATP binding cassette transporter A1 in RAW cells. Infect. Immun. 2002, 70, 2995-3003.

Sample Availability: Samples of the compounds are available from the authors and commercially available.

(C) 2011 by the authors; licensee MDPI, Basel, Switzerland. This article is an open access article distributed under the terms and conditions of the Creative Commons Attribution license (http://creativecommons.org/licenses/by/3.0/). 